\title{
INFORME BIBLIOMÉTRICO
}

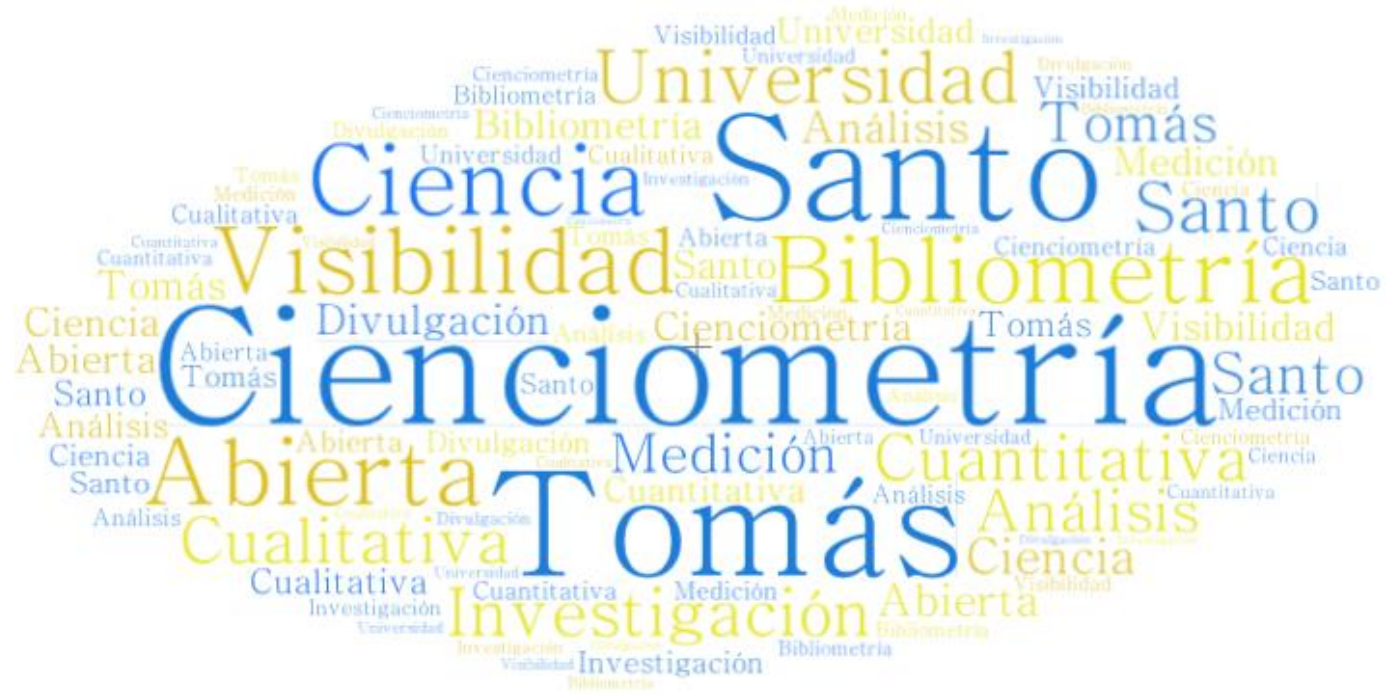

Observatorio de cienciometría USTA

Dirección de Investigación e Innovación - Sede principal

Centro de Recursos para el Aprendizaje y la Investigación CRAIUSTA

Por: Camilo A. Corchuelo R. y Luz Marina Paez

DOI: 10.13140/RG.2.2.31403.59687 


\section{Revisión para publicación}

\section{RESUMEN}

Informe de caracterización de tema "The study of the socio-ecological systems and the elites of knowledge, framework applicable to the Colombia case" para publicación en revistas indexadas en corriente principal y alterna en el marco internacional y el Sistema Nacional De Ciencia, Tecnología e Innovación de Colciencias. Este es un insumo para la comunidad académica USTA cuyo objetivo es publicar con pertinencia y coherencia en revistas en Bases de Datos Comprensivas, Sistemas de Indexación y Resumen -SIR, Directorios, entre otros.

Palabras Claves: Bibliometría, cienciometría, revistas, visibilidad académica, scopus, wos, biodiversidad; grupos étnicos; sistemas socioecológicos; sociedades del conocimiento.

\section{ABSTRACT}

Characterization report on the topic "The study of the socio-ecological systems and the elites of knowledge, framework applicable to the Colombian case" for publication in journals indexed in mainstream and alternating current in the international framework and the National Science, Technology and Innovation System of Colciencias. This is an input for the USTA academic community whose objective is to publish with relevance and coherence in journals in Comprehensive Databases, Indexing Systems and Summary -SIR, Directories, among others.

Keywords: Bibliometric, cienciometric, journal, academic visibility, scopus, wos, biodiversity, ethnic groups, social-ecological systems, knowledge societies, ecological assessment, social-ecological, social-ecological systems, ethnic groups. 


\section{Revisión para publicación}

\section{TABLA DE

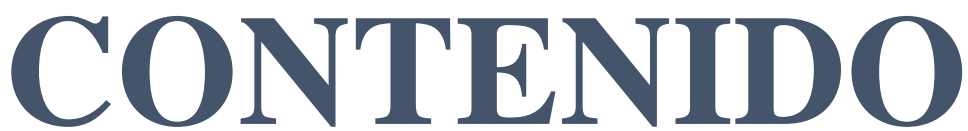

RESUMEN

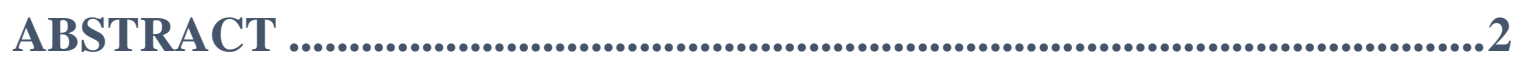

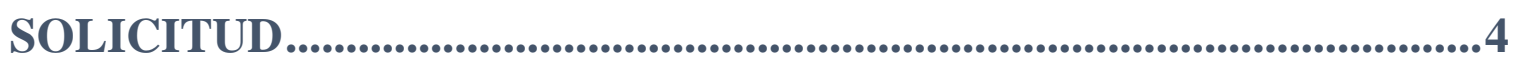

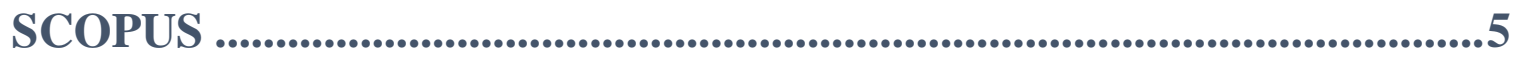

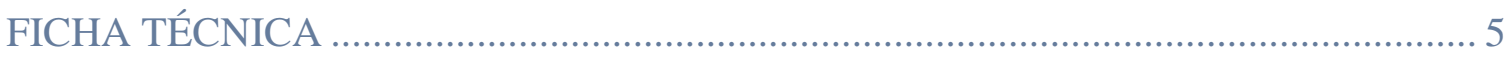

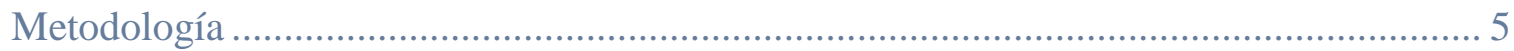

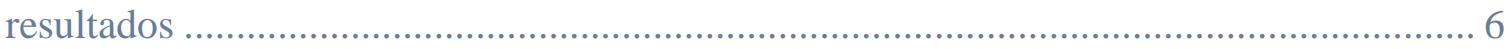

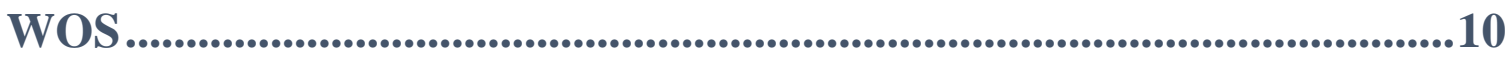

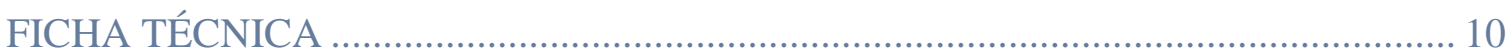

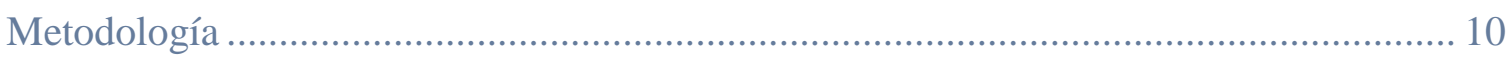

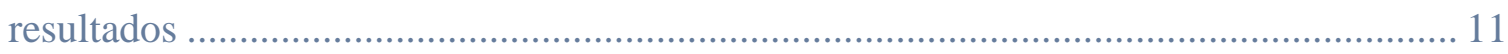

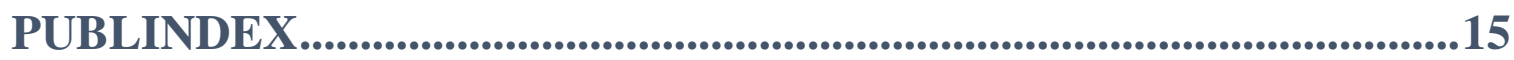

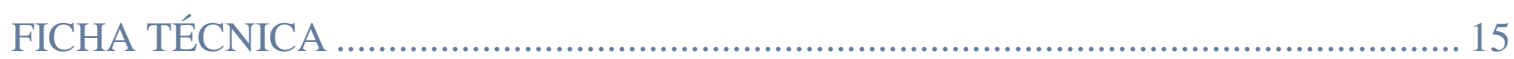

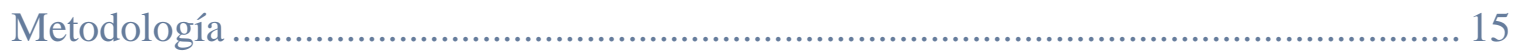

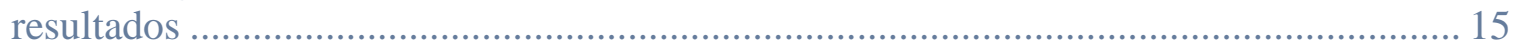

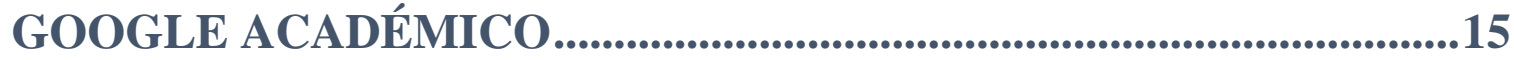

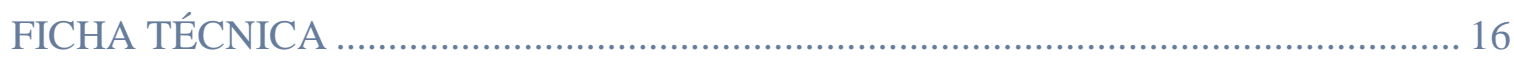

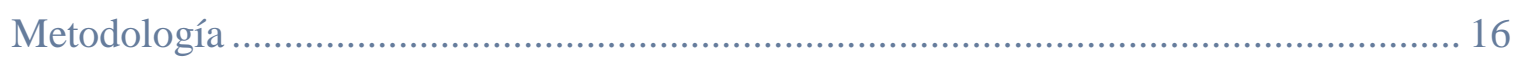

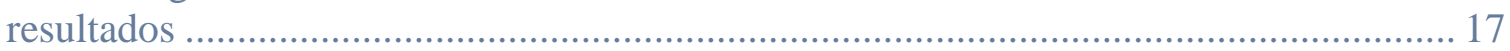

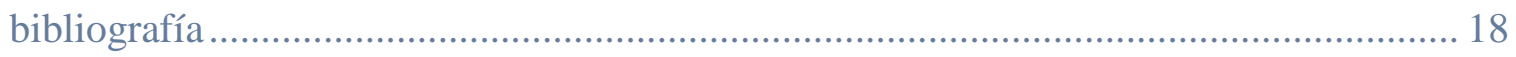




\title{
Revisión para publicación
}

\section{SOLICITUD}

Estimados compañeros

Estoy buscando una publicación adecuada para el artículo:

"THE STUDY OF THE SOCIO-ECOLOGICAL SYSTEMS AND THE ELITES OF KNOWLEDGE, FRAMEWORK APPLICABLE TO THE COLOMBIAN CASE"

KEYWORDS: Biodiversity; Ethnic groups; Social-ecological Systems; knowledge societies

\begin{abstract}
This study aims to analyze the background for studying of socio-ecological systems that may apply to the Rancheria River basin. It includes a systematic review of both theoretical and methodological tools based on case studies involving regions of the different places on the world. First, the literature from specialized databases and repositories was identified. Then, the case studies that were applied to specific problems of socio-ecological systems were selected and analyzed to determine useful methodologies for Colombia.
\end{abstract}




\section{Revisión para publicación}

\section{SCOPUS}

\section{FICHA TÉCNICA}

\begin{tabular}{|l|l|}
\hline & \multicolumn{1}{|c|}{ Expresión de búsqueda } \\
\hline $\begin{array}{l}\text { Estrategia de } \\
\text { Búsqueda }\end{array}$ & $\begin{array}{l}\text { Biodiversity ECOLOGICAL assessment Social-ecological Systems Ethnic groups } \\
\text { knowledge societies }\end{array}$ \\
\hline Tesauro & Environmental Thesaurus \\
\hline Período de análisis: $2015-2020$ \\
\hline Herramientas: VandagePoint, Microsoft Excel \\
\hline Fecha de consulta: $17 / 02 / 2020$ \\
\hline
\end{tabular}

\section{METODOLOGÍA}

Búsqueda de

información

(Ecuaciones de

búsqueda)
Depuración datos

(López-López, 1996)
Análisis de datos 


\section{Revisión para publicación}

\section{RESULTADOS}

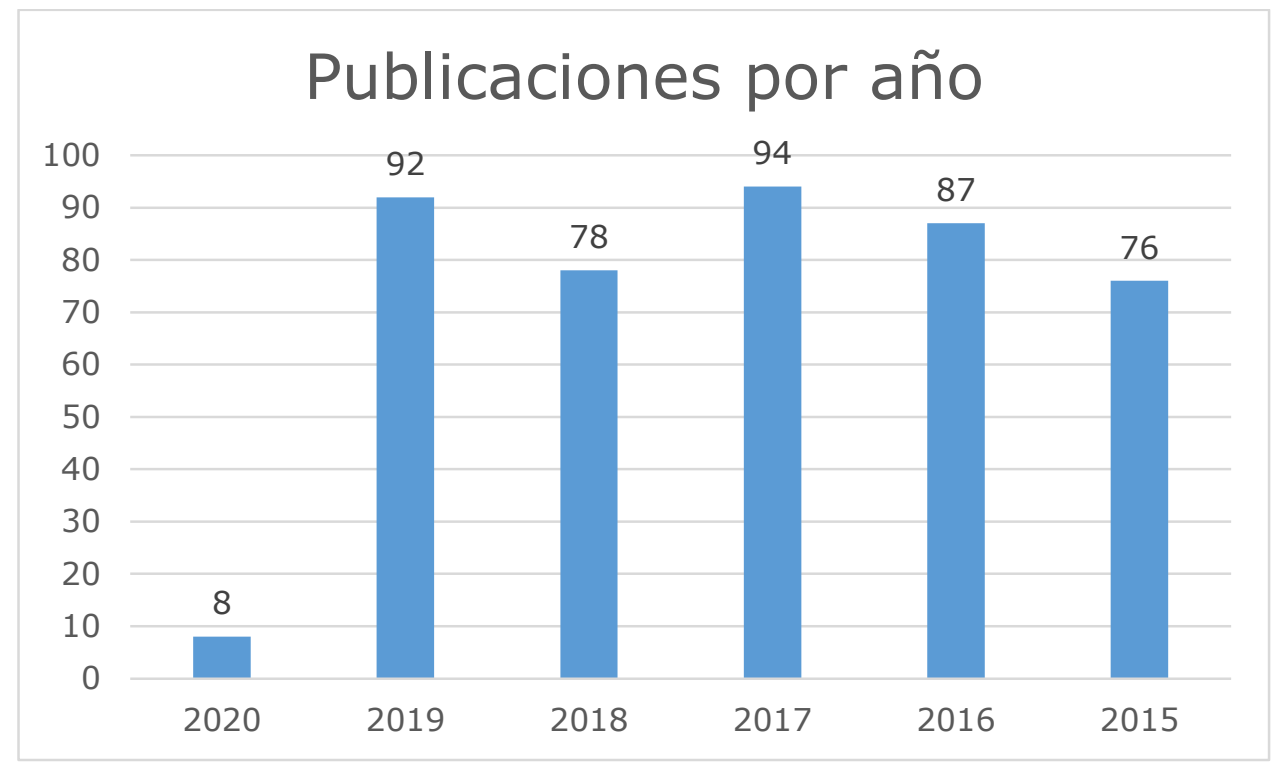

Gráfico 1. Artículos por año (López-López, 1996). Elaboración propia. Fuente: Scopus

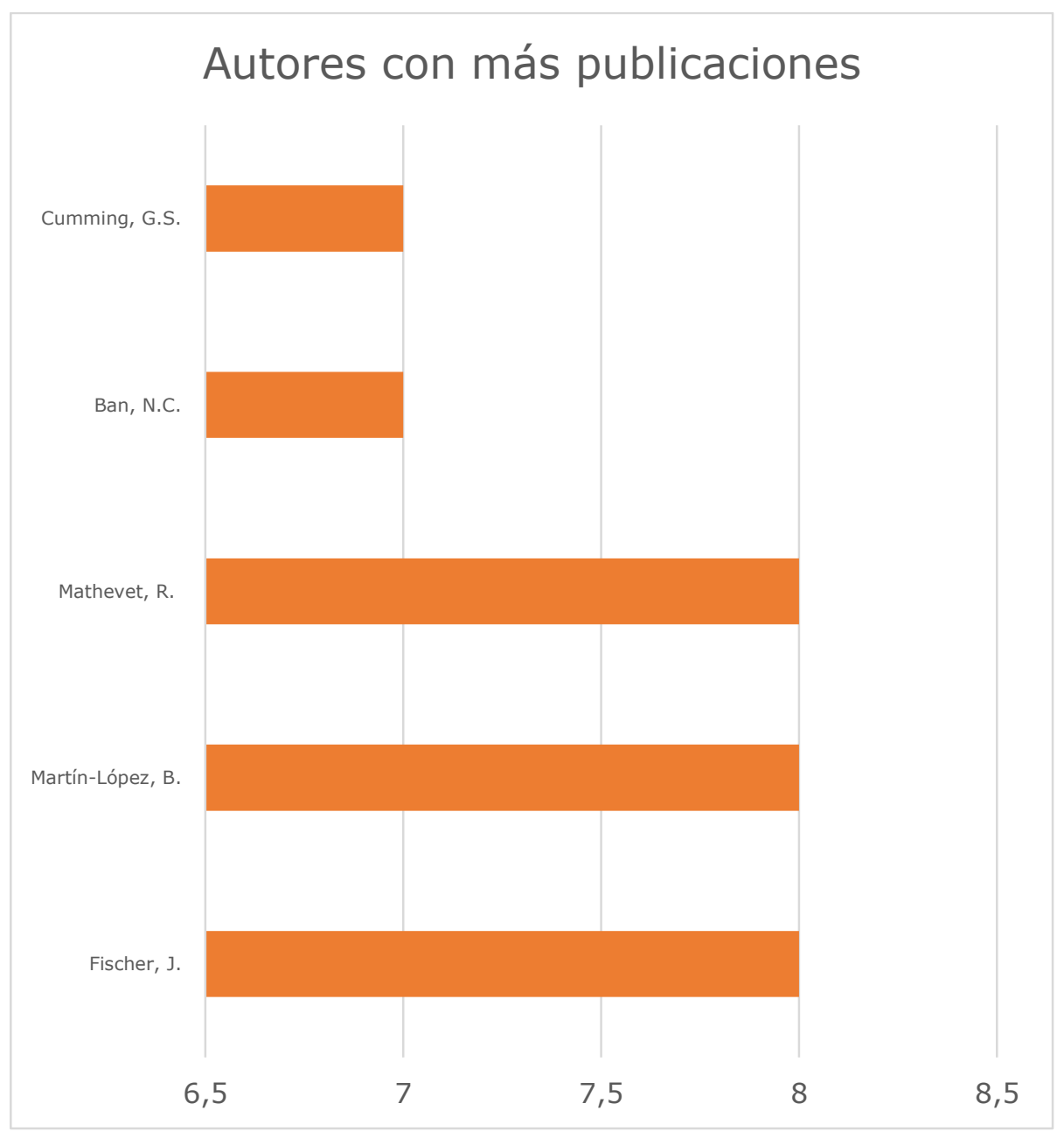

Gráfico 2. Autores con más publicaciones. Elaboración propia. Fuente: Scopus 


\section{Revisión para publicación}

\section{Conservation of Natural Resources}

governance approach ethnic group Animals
adaptive management ${ }_{\text {Land us }}$

\section{environmental protection}

Biodiversity wastrabutity

ecosystem service conservation management

Ecology Climate change

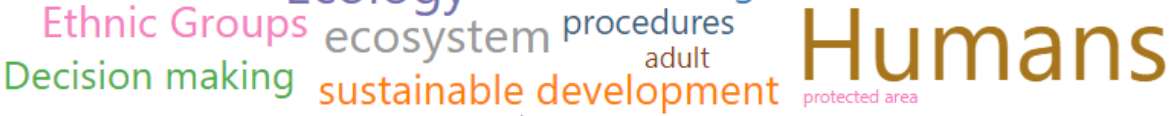

nonhuman priority journal male

Gráfico 3. Palabras claves con mayor concurrencia (mayor a 20). Elaboración propia. Fuente: Scopus 


\section{Revisión para publicación}

- Cienciometríasant

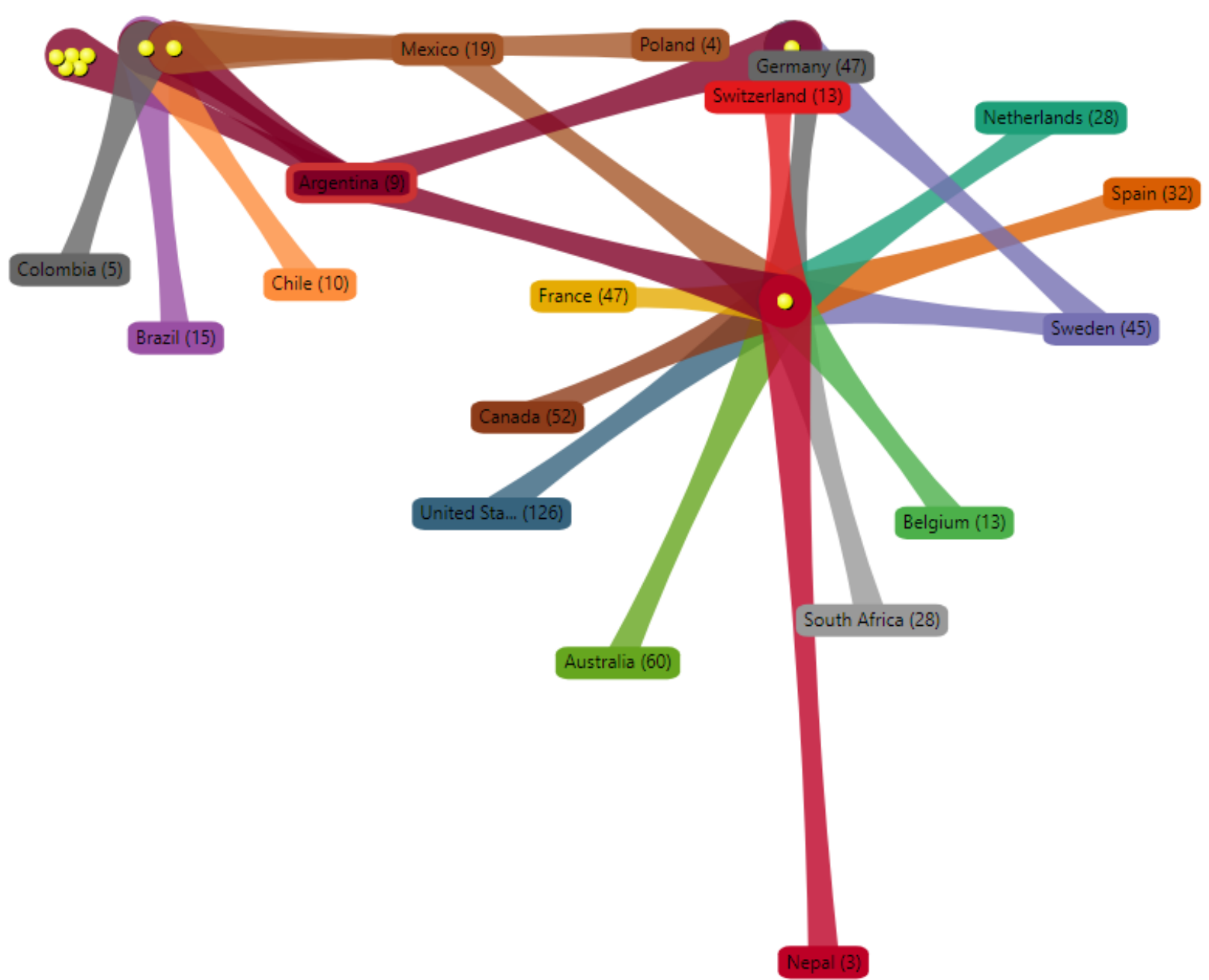

Gráfico 4. Mapa de relación del país de filiación de los autores. Fuente: Scopus 


\section{Revisión para publicación}

A continuación, se listan las revistas indexadas que han realizado publicaciones en el tema. Por tal motivo, tienen una alta probabilidad de recibir el artículo.

Tabla1. Revistas indexadas en Scopus con publicaciones en el tema por cuartil.

\begin{tabular}{|l|c|}
\hline \multicolumn{1}{|c|}{ Revista } & Cuartil \\
\hline Ecology and Society & $\underline{\mathrm{Q} 1}$ \\
\hline Journal of Ethnobiology and Ethnomedicine & $\underline{\mathrm{Q}}$ \\
\hline Sustainabilityopen access & $\underline{\mathrm{Q} 1}$ \\
\hline PLoS ONE & $\underline{\mathrm{Q}}$ \\
\hline Conservation Biology & $\underline{\mathrm{Q}}$ \\
\hline Ecosystem Services & $\underline{\mathrm{Q}}$ \\
\hline Land Use Policy & $\underline{\mathrm{Q}}$ \\
\hline Regional Environmental Change & $\underline{\mathrm{Q}}$ \\
\hline Journal of Environmental Management & \\
\hline
\end{tabular}

Elaboración propia. Fuente: Scopus 


\section{Revisión para publicación}

\section{WOS}

\section{FICHA TÉCNICA}

\begin{tabular}{|l|l|}
\hline & \multicolumn{1}{|c|}{ Expresión de búsqueda } \\
\hline Estrategia de & (TS=( biodiversity OR "ECOLOGICAL assessment" AND "Social- \\
ecological" OR "Social-ecological Systems" OR "Ethnic groups" OR \\
& "knowledge societies")) AND TIPOS DE DOCUMENTOS: (Article) \\
& Refinado por: AÑOS DE PUBLICACIÓN: ( 2020 OR 2015 OR 2019 OR \\
& 2018 OR 2017 OR 2016 ) AND TIPOS DE DOCUMENTOS: (ARTICLE \\
& Período de tiempo: Todos los años. Índices: SCI-EXPANDED, SSCI, \\
& A\&HCI, ESCI. \\
\hline Tesauro & Environmental Thesaurus \\
\hline Período de análisis: 2015-2020 \\
\hline Herramientas: VandagePoint, Microsoft Excel \\
\hline Fecha de consulta: 15/2/2020 \\
\hline
\end{tabular}

\section{METODOLOGÍA}

Búsqueda de información

(Ecuaciones de búsqueda)
Depuración datos

(López-López, 1996) 


\section{Revisión para publicación}

\section{RESULTADOS}

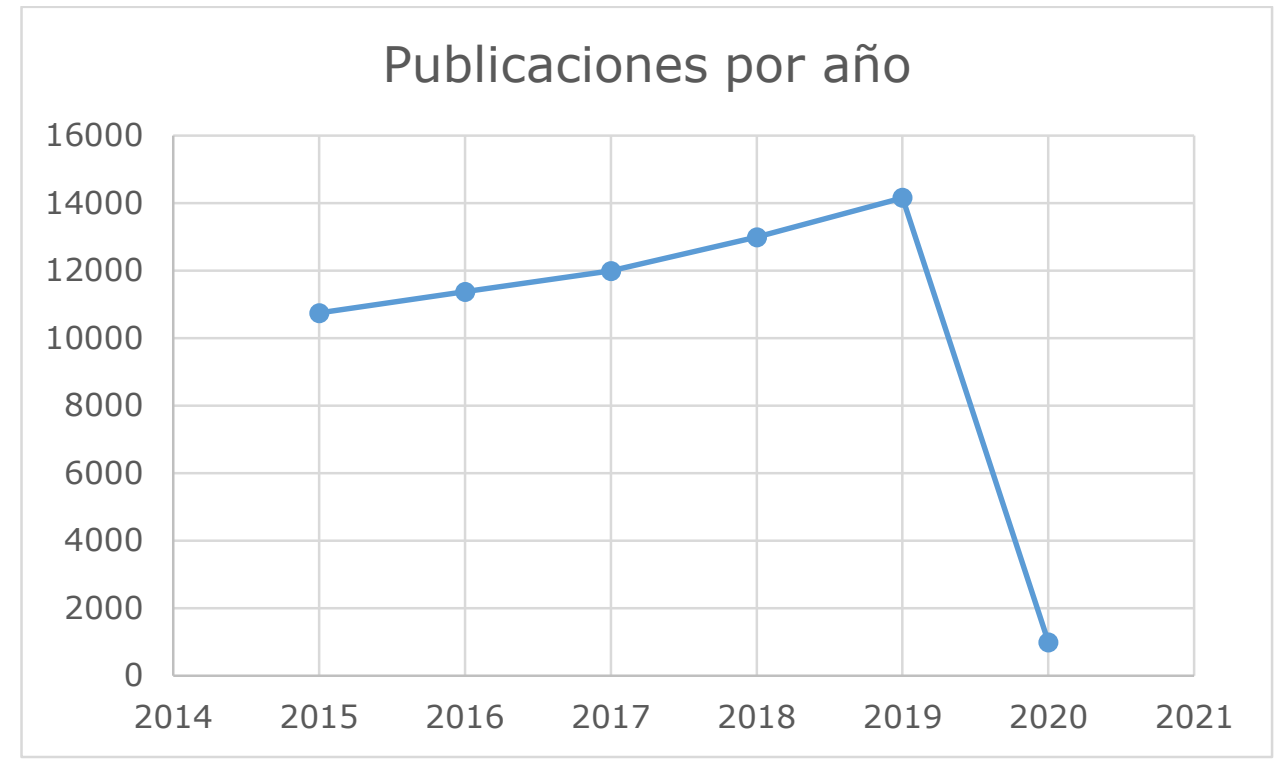

Gráfico 4. Artículos por año (López-López, 1996). Elaboración propia. Fuente: WOS

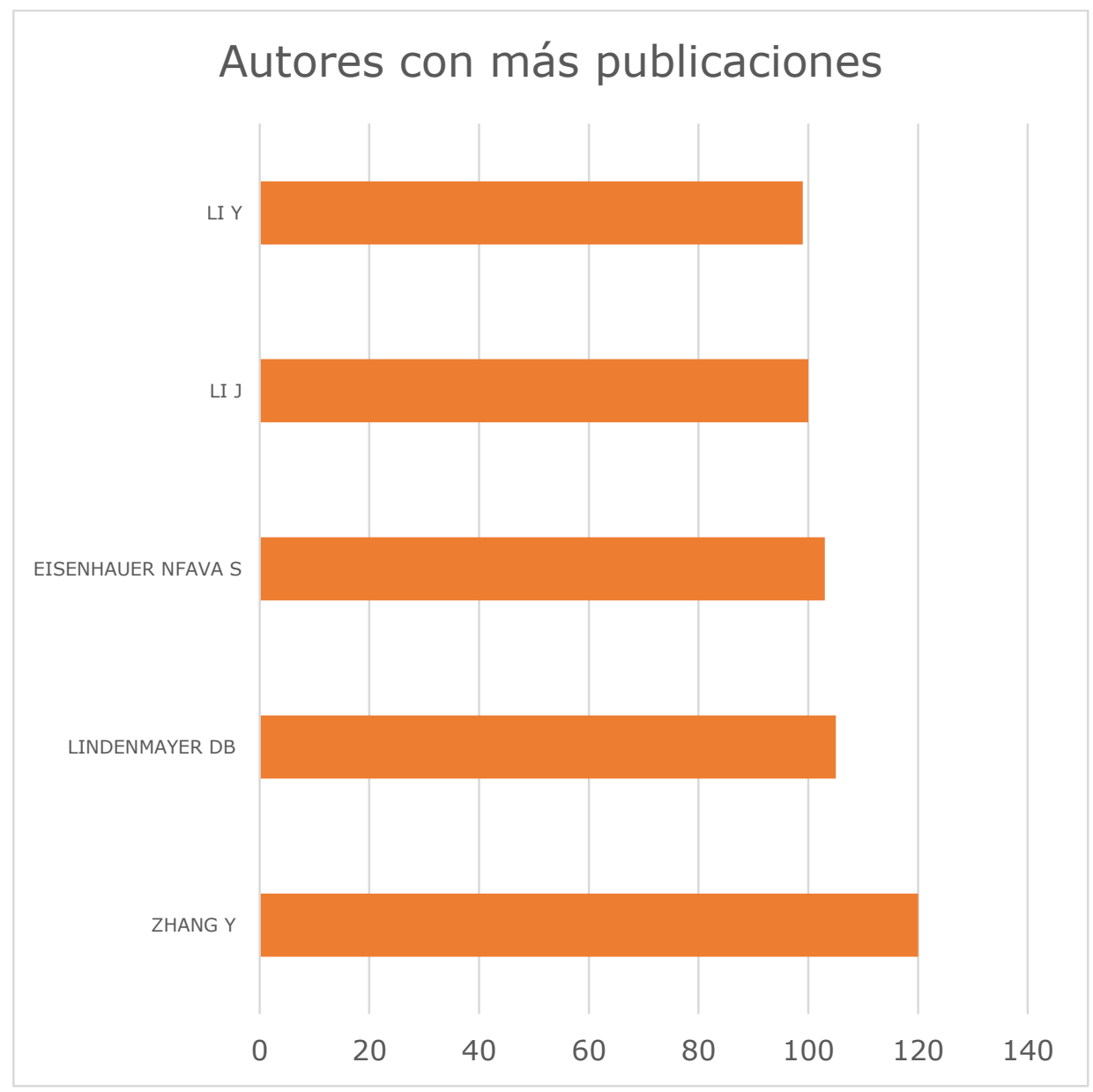

Gráfico 5. Autores con más publicaciones. Elaboración propia. Fuente: WOS 


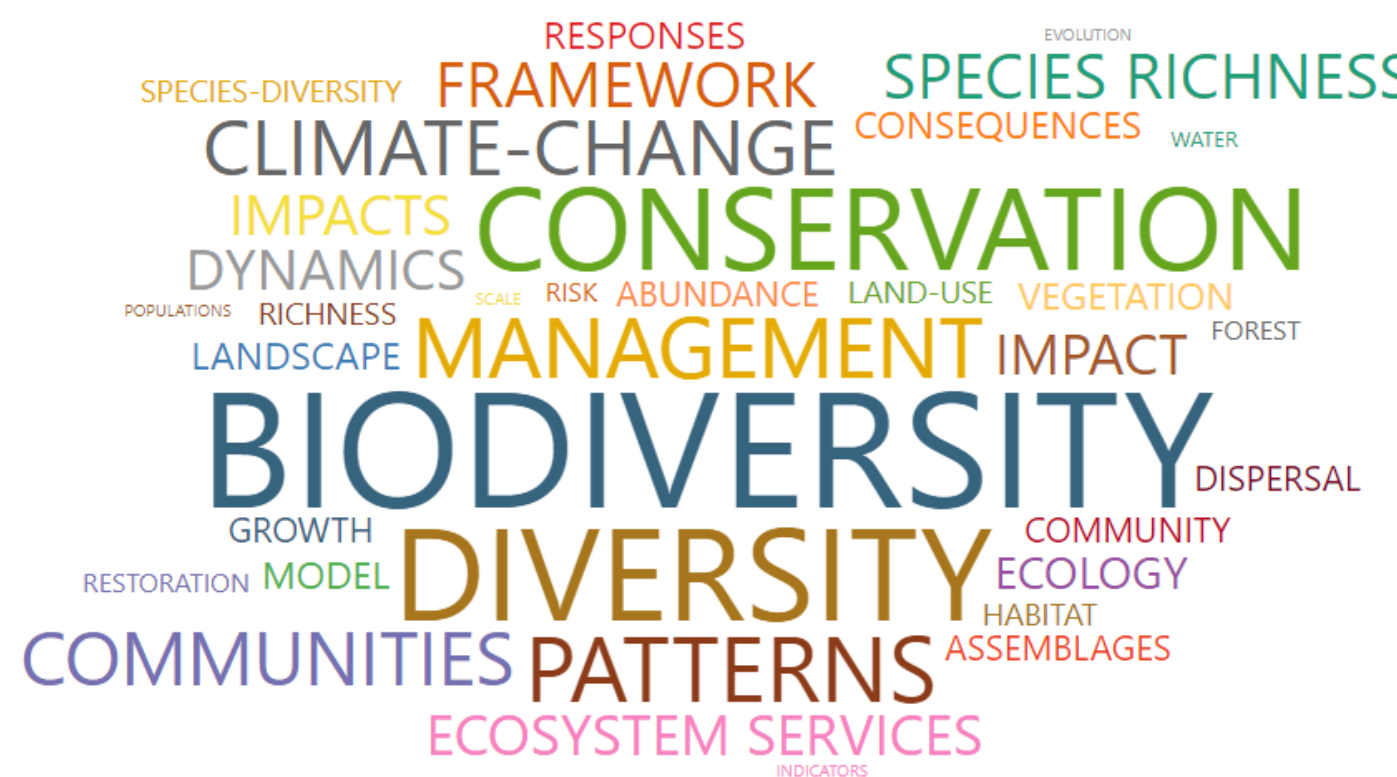

Gráfico 6. Palabras claves con mayor concurrencia (mayor a 10). Elaboración propia. Fuente: WOS 


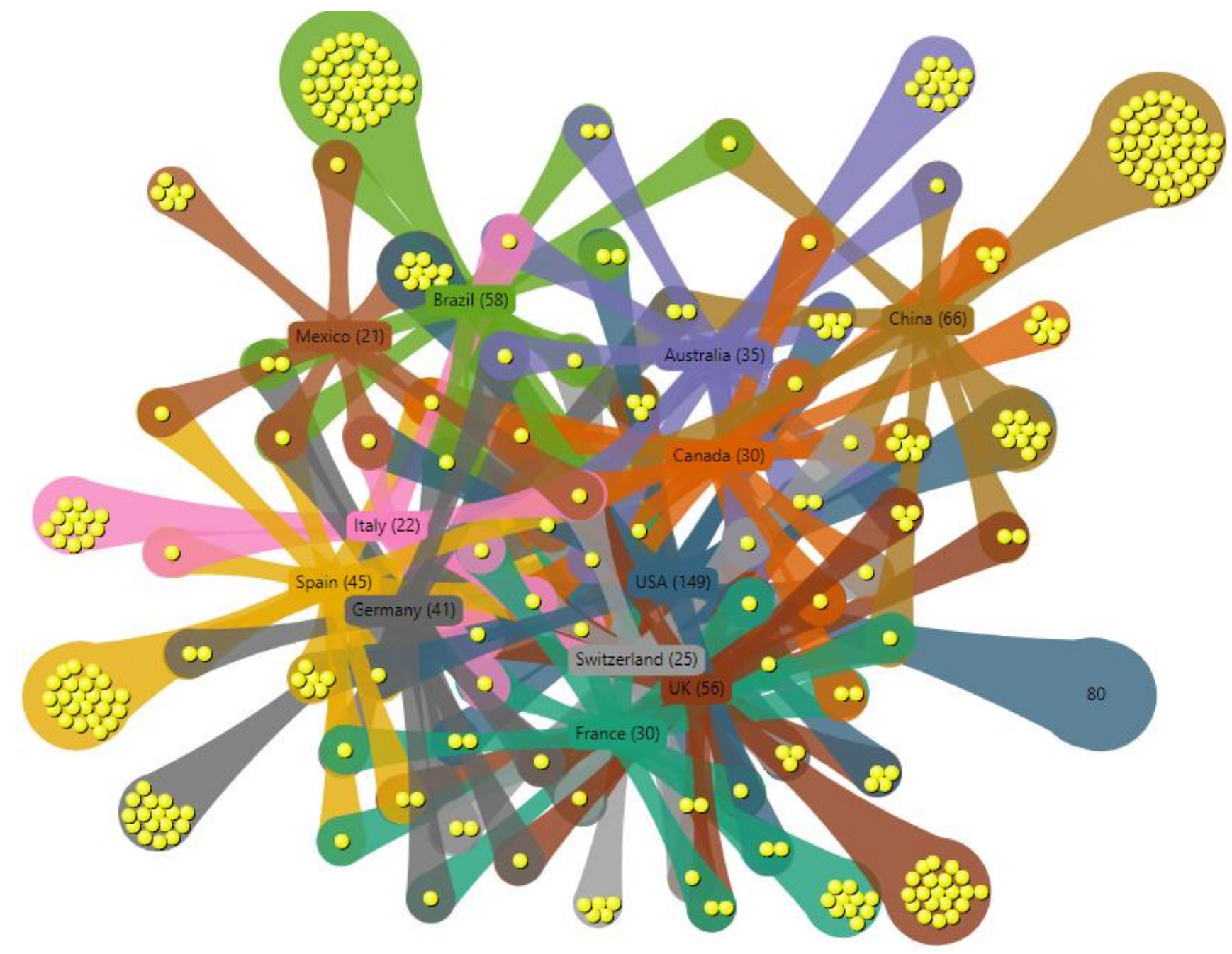

Mapa de relación del país de filiación de los autores. Fuente: WOS 


\section{Revisión para publicación}

A continuación, se listan las revistas indexadas que han realizado publicaciones en el tema. Por tal motivo, tienen una alta probabilidad de recibir el artículo.

Tabla1. Revistas indexadas en WOS con publicaciones en el tema por cuartil.

\begin{tabular}{|l|c|}
\hline \multicolumn{1}{|c|}{ Revista } & Cuartil \\
\hline ECOLOGICAL INDICATORS & Q1 \\
\hline SCIENCE OF THE TOTAL ENVIRONMENT & Q1 \\
\hline FOREST ECOLOGY AND MANAGEMENT & Q1 \\
\hline JOURNAL OF BIOGEOGRAPHY & Q1 \\
\hline ECOLOGY AND EVOLUTION & Q1 \\
\hline POLISH JOURNAL OF ENVIRONMENTAL STUDIES & Q2 \\
\hline
\end{tabular}

Elaboración propia. Fuente: WOS 


\section{Revisión para publicación}

\section{PUBLINDEX}

FICHA TÉCNICA

\begin{tabular}{|l|l|}
\hline \multicolumn{1}{|c|}{ Fuente } & Publindex ( Convocatoria 830 de 2018) \\
\hline Herramientas: Microsoft Excel \\
\hline Fecha de consulta: $15 / 02 / 2020$ \\
\hline
\end{tabular}

\section{METODOLOGÍA}

\section{RESULTADOS}

Revistas en el área que podrían recibir el artículo (Colciencias, 2016, 2018).

Tabla 2. Revistas Publindex en el área de conocimiento.

\begin{tabular}{|l|l|c|c|}
\hline \multicolumn{1}{|c|}{ Revista } & \multicolumn{1}{|c|}{ Institución } & Categoría & $\begin{array}{c}\text { Homologación } \\
\text { en el modelo } \\
\text { de Colciencias }\end{array}$ \\
\hline CALDASIA & $\begin{array}{l}\text { UNIVERSIDAD NACIONAL DE } \\
\text { COLOMBIA }\end{array}$ & Q & Q3 \\
\hline Acta Biológica Colombiana & $\begin{array}{l}\text { UNIVERSIDAD NACIONAL DE } \\
\text { COLOMBIA }\end{array}$ & B & Q3 \\
\hline CIENCIA EN DESARROLLO & $\begin{array}{l}\text { UNIVERSIDAD PEDAGÓGICA Y } \\
\text { TECNOLÓGICA DE COLOMBIA }\end{array}$ & $\mathrm{C}$ & $\mathrm{Q} 4$ \\
\hline $\begin{array}{l}\text { EARTH SCIENCES RESEARCH } \\
\text { JOURNAL }\end{array}$ & $\begin{array}{l}\text { UNIVERSIDAD NACIONAL DE } \\
\text { COLOMBIA }\end{array}$ & B & Q3 \\
\hline BIOTA COLOMBIANA & $\begin{array}{l}\text { INSTITUTO DE INVESTIGACIÓN DE } \\
\text { RECURSOS BIOLÓGICOS } \\
\text { ALEXANDER VON HUMBOLDT }\end{array}$ & $\mathrm{C}$ & Q4 \\
\hline Ambiente y Desarrollo & $\begin{array}{l}\text { PONTIFICIA UNIVERSIDAD } \\
\text { JAVERIANA }\end{array}$ & $\mathrm{C}$ & Q4 \\
\hline
\end{tabular}

Elaboración propia. Fuente: Publindex (Corchuelo-Rodríguez et al., 2020) 


\section{Revisión para publicación}

\section{GOOGLE ACADÉMICO}

\section{FICHA TÉCNICA}

\begin{tabular}{|l|l|}
\hline & \multicolumn{1}{|c|}{ Expresión de búsqueda } \\
\hline $\begin{array}{l}\text { Estrategia de } \\
\text { Búsqueda }\end{array}$ & $\begin{array}{l}\text { ( biodiversity OR "ECOLOGICAL assessment" AND "Social-ecological" } \\
\text { OR "Social-ecological Systems" OR "Ethnic groups" OR "knowledge } \\
\text { societies" ) }\end{array}$ \\
\hline Tesauro & Environmental Thesaurus \\
\hline Período de análisis: 2015-2020 \\
\hline Herramientas: Harzing's Publish or Perish 6.21, Microsoft Excel \\
\hline Fecha de consulta: 15/2/2020 \\
\hline
\end{tabular}

\section{METODOLOGÍA}




\section{Revisión para publicación}

\section{RESULTADOS}

Tabla 3. Top 10 de las publicaciones con más citas.

\begin{tabular}{|c|c|c|c|}
\hline Cites & Authors & Title & Year \\
\hline 480 & $\begin{array}{l}\text { AD Guerry, S Polasky, J } \\
\text { Lubchenco... }\end{array}$ & $\begin{array}{l}\text { Natural capital and ecosystem services informing decisions: From } \\
\text { promise to practice }\end{array}$ & 2015 \\
\hline 465 & $\begin{array}{l}\text { U Pascual, P Balvanera, S } \\
\text { Díaz, G Pataki... }\end{array}$ & Valuing nature's contributions to people: the IPBES approach & 2017 \\
\hline 366 & $\begin{array}{l}\text { EM Bennett, W Cramer, } \\
\text { A Begossi, G Cundill... }\end{array}$ & $\begin{array}{l}\text { Linking biodiversity, ecosystem services, and human well-being: three } \\
\text { challenges for designing research for sustainability }\end{array}$ & 2015 \\
\hline 345 & S Molnar & Human variation: races, types, and ethnic groups & 2015 \\
\hline 337 & $\begin{array}{l}\text { RW Mackelprang, RO } \\
\text { Salsgiver }\end{array}$ & Disability: A diversity model approach in human service practice & 2016 \\
\hline 294 & $\begin{array}{l}\text { DJ Abson, J Fischer, J } \\
\text { Leventon, J Newig... }\end{array}$ & Leverage points for sustainability transformation & 2017 \\
\hline 274 & $\begin{array}{l}\text { C Folke, R Biggs, AV } \\
\text { Norström, B Reyers... }\end{array}$ & Social-ecological resilience and biosphere-based sustainability science & 2016 \\
\hline 263 & C Folke & Resilience (republished) & 2016 \\
\hline 256 & $\begin{array}{l}\text { JA Oldekop, G Holmes, } \\
\text { WE Harris... }\end{array}$ & $\begin{array}{l}\text { A global assessment of the social and conservation outcomes of } \\
\text { protected areas }\end{array}$ & 2016 \\
\hline 230 & $\begin{array}{l}\text { RD Long, A Charles, RL } \\
\text { Stephenson }\end{array}$ & Key principles of marine ecosystem-based management & 2015 \\
\hline
\end{tabular}

A continuación, se listan las revistas indexadas (top 5) de los artículos con más citas entre 2015-2020. Por tal motivo, tienen una alta probabilidad de recibir el artículo.

Tabla 4. Revistas indexadas en Google académico con más publicaciones en el tema.

\begin{tabular}{|l|l|l|l|}
\hline \multicolumn{1}{|c|}{ Revista } & \multicolumn{1}{c|}{ Scopus } & \multicolumn{1}{c|}{ WOS } & \multicolumn{1}{c|}{$\begin{array}{c}\text { Categoría en } \\
\text { Colciencias }\end{array}$} \\
\hline Ecology and Society & SI & SI & Tipo A1 \\
\hline Current Opinion in Biotechnology & SI & SI & Tipo A1 \\
\hline Business Process Management Journal & SI & SI & Tipo A1 \\
\hline Marine Policy & SI & SI & Tipo A1 \\
\hline Ecosystem Services & SI & SI & Tipo A1 \\
\hline
\end{tabular}

Elaboración propia. Fuente: Publindex 


\section{Revisión para publicación}

\section{BIBLIOGRAFÍA}

Colciencias. (2016). Convocatoria para Indexación de Revistas Científicas Colombianas Especializadas-Publindex (No. 768 de 2016).

https://www.colciencias.gov.co/convocatorias/investigacion/convocatoria-paraindexacion-revistas-cientificas-colombianas

Colciencias. (2018). Convocatoria Nacional para el reconocimiento y medición de grupos de investigación, desarrollo tecnológico o de innovación y para el reconocimiento de investigadores del Sistema Nacional de Ciencia, Tecnología e Innovación - SNCTeI, 2018.

https://www.colciencias.gov.co/sites/default/files/4._anexo_1._documento_concep tual_del_modelo_de_reconocimiento_y_medicion_de_grupos_de_investigacion_2 018.pdf

Corchuelo-Rodríguez, C. A., Patacón-Ruiz, I., \& Piza-Amado, K. (2020). Revistas de Ciencias Sociales en el modelo de clasificación de Publindex: Prospectiva de la convocatoria n. ${ }^{\circ} 830$ del 2018 en la fase III. Impacto de la revista científica. Revista Interamericana de Investigación, Educación y Pedagogía, RIIEP, 13(1), 137-155.

Dueñas, D. C., Rodriguez, C. A. C., \& Ruiz, I. P. P. (2019). Revistas de ciencias sociales y el derecho en el modelo de clasificación de Publindex: Impacto de la revista científica. Verba luris, 42, 113-121. https://doi.org/10.18041/01213474/verbaiuris. 42.5662

López-López, P. (1996). Introducción a la bibliometría. Promolibro. 